\title{
Active vibration control (AVC) of a satellite boom structure using optimally positioned stacked piezoelectric actuators
}

\author{
M. Moshrefi-Torbati ${ }^{\mathrm{a}, *}$, A.J. Keane ${ }^{\mathrm{b}}$, S.J. Elliott ${ }^{\mathrm{c}}$, M.J. Brennan ${ }^{\mathrm{c}}$, \\ D.K. Anthony ${ }^{\mathrm{c}}, \mathrm{E}$. Rogers ${ }^{\mathrm{d}}$ \\ ${ }^{a}$ Research Institute for Industry, School of Engineering Sciences, University of Southampton, Southampton, UK \\ ${ }^{\mathrm{b}}$ Computational Engineering and Design Group, School of Engineering Sciences, University of Southampton, \\ Southampton, UK \\ ${ }^{\mathrm{c}}$ Institute of Sound and Vibration Research, University of Southampton, Southampton, UK \\ ${ }^{\mathrm{d}}$ School of Electronics and Computer Science, University of Southampton, Southampton, UK
}

Received 3 October 2002; received in revised form 25 July 2005; accepted 26 July 2005

Available online 5 October 2005

\begin{abstract}
In this paper, results for active vibration control predicted from experimental measurements on a lightweight structure are compared with purely computational predictions. The structure studied is a $4.5 \mathrm{~m}$ long satellite boom consisting of 10 identical bays with equilateral triangular cross sections. First, the results from a Fortran code that is based on a receptance analysis are validated against the experimental forced response of the boom structure. Exhaustive searches are then carried out to find the optimum positions for one and two actuators. Finally, a genetic algorithm is employed to find high-quality positions for three actuators on the structure that will achieve the greatest reductions in vibration transmission. Having found these actuator positions, experiments are then carried out to verify the quality of the theoretical predictions. It was found that the attenuation achievable in practice for one, two and three actuators were, respectively, 15.1, 26.1 and $33.5 \mathrm{~dB}$.
\end{abstract}

(C) 2005 Elsevier Ltd. All rights reserved.

\footnotetext{
*Corresponding author. Tel.: + 442380598583 ; fax: + 442380597051.

E-mail addresses: m.m.torbati@soton.ac.uk (M. Moshrefi-Torbati), ajk@soton.ac.uk (A.J. Keane), sje@isvr.soton.ac.uk (S.J. Elliott), mjb@isvr.soton.ac.uk (M.J. Brennan), dka@soton.ac.uk (D.K. Anthony), etar@ecs.soton.ac.uk (E. Rogers).
} 


\begin{tabular}{|c|c|c|c|}
\hline \multicolumn{2}{|c|}{ Nomenclature } & $J_{\min }$ & minimum value of the cost function \\
\hline $\begin{array}{l}\mathbf{v} \\
f_{p}\end{array}$ & $\begin{array}{l}\text { net velocity vector } \\
\text { primary force }\end{array}$ & $\alpha$ & $\begin{array}{l}\text { frequency-averaged attenuation in dec- } \\
\text { ibels }\end{array}$ \\
\hline $\mathbf{f}_{s}$ & control forces (controlled by the AVC) & $\mathbf{v}_{p}$ & velocities at the end three joints (i.e., \\
\hline Y & transformed mobility transfer matrix & & joints 31,32 and 33 ) resulting from \\
\hline $\mathbf{U}$ & the mobility matrix & & primary excitation \\
\hline $\mathbf{T}$ & the transformation matrix & $\mathbf{v}_{s}$ & velocities resulting from the active \\
\hline$J$ & quadratic objective function & & vibration control in the absence of \\
\hline$\Delta \omega$ & frequency increment & & primary excitation \\
\hline$\omega 1$ & lower frequency & $q_{s}$ & total control effort required by the feed- \\
\hline$\overline{J_{\text {nom }}}$ & $\begin{array}{l}\text { average value of the cost function } \\
\text { without active vibration control (AVC) }\end{array}$ & & $\begin{array}{l}\text { forward control system across the } \\
\text { frequency band }\end{array}$ \\
\hline
\end{tabular}

\section{Introduction}

The vibration and noise control problems that arise in many engineering projects are particularly severe when lightweight structures are employed. This is an area where many traditional techniques have been tried with relatively little success. Perhaps the most challenging vibration control issues arise in the design of space systems that involve satellites with highly sensitive instrumentation packages. To function correctly these packages must be supported on structures, where the vibration levels have been reduced to extremely low levels (i.e., microvibrations). This need becomes more severe when the instrument concerned forms one of the individual sensors of a multi-sensor interferometric telescope or synthetic aperture radar [1]. In such cases there is a need to support instruments spaced tens of metres apart using structural booms, with the relative motions between their ends being restricted to microns over wide ranges of excitation frequency [2].

A number of design approaches have been proposed to try to meet these demanding requirements but it is still not clear how best to proceed in this field [3]. The most common treatment for such problems is to use anti-vibration mountings or to coat the structural elements with viscoelastic damping materials with consequent weight and cost penalties. Moreover, the effectiveness of such treatments diminishes at low vibration levels, which makes continuously improving noise and vibration targets difficult to meet. Clearly, if the vibrational energy could be contained near the points of excitation there would be a reduced need for damping treatments and, additionally, they could be concentrated in regions where they were most effective.

This is precisely the aim of the vibration isolators used between most pieces of equipment and their supporting structure. However, such isolators cannot deliver the desired behaviour in all situations, particularly for sensitive equipment. Thus, there is the need for some kind of widely applicable, generic structural filter design capability that can be used to build desirable characteristics into a structure, retaining its ability to carry static loads while blocking higher frequency motions. To gain maximum benefit from the available technologies such a capability 
would ideally be based on an integrated active/passive approach, with these two techniques being used in tandem and together tackling the widest possible range of excitation frequencies.

This paper reflects part of a research programme currently being undertaken into the development of a vibration control approach for such systems. Here, the focus is on controlling a full three-dimensional truss structure by using stacked piezoelectric actuators. In the more complex cases, the optimal actuator positions are found by the use of an evolutionary optimization method, namely a genetic algorithm (GA). In the optimizations studied here the search space consists of a discrete set of solutions, i.e., over the large number of possible actuator position combinations. The maximum reduction of the vibration transmission for each combination of actuators is partly dependent on the mechanical coupling between the actuators and the region of concern. To find the best combinatorial positions, candidates are ranked based on their performance.

The structure used in this study consists of 93 individual beams and allows for bending, axial and torsional vibrations. The receptance analysis used here has already been fully validated against detailed finite element (FE) models of the structures carried out with commercial FE codes [4]. In order to quantify the usual inherent modelling uncertainties that arise when working in this field, and based on the theoretical designs produced, a series of experiments have been performed. An initial base-line structure of $4.5 \mathrm{~m}$ length with 10 identical bays (representative of a satellite boom) was first constructed. The response of this structure, when suspended in the laboratory, was measured and its experimental forced response compared with the theoretical predictions. In the experiments, the undesirable noise disturbances that the boom would experience in practice are represented as a random transverse force at one of the joints in the structure. Optimum positions for one, two and three actuators are found computationally using in-house developed software [5] and experiments for one and three actuators are carried out. Although, the investigation presented in this paper is based on satellite structures, the overall results may be of interest in all fields where reduced vibration transmission is important, such as aircraft and car design.

\section{The structure}

Figs. 1 and 2 illustrate the geometry of the boom structure and the numbering systems used for the joints and beam elements, respectively. All the 93 Euler-Bernoulli beams in the structure have the same properties per unit length. They have an axial rigidity $E A$ of $2.184 \mathrm{MN}$, a bending rigidity $E I$ of $5.503 \mathrm{~N} \mathrm{~m}^{2}$ and mass per unit length of $0.085 \mathrm{~kg} / \mathrm{m}$. The beams are all either $0.450 \mathrm{~m}$ or $0.636 \mathrm{~m}$ long and are joined together by 33 aluminium spheres of $25 \mathrm{~mm}$ diameter. The model was excited by a point transverse force on the fourth joint, $0.45,0.0$, $0.0 \mathrm{~m}$. Based on initial trial experiments, a value of 0.005 was used for the structural viscous damping ratio in all computations. During optimization, the main goal is to minimize the mean vibrational energy level in the right-hand three joints, 31, 32 and 33 (which in practice might be attached to an instrumentation package) between 150 and $250 \mathrm{~Hz}$. This spans a frequency range where conventional approaches to satellite boom vibration control are currently not very effective. 


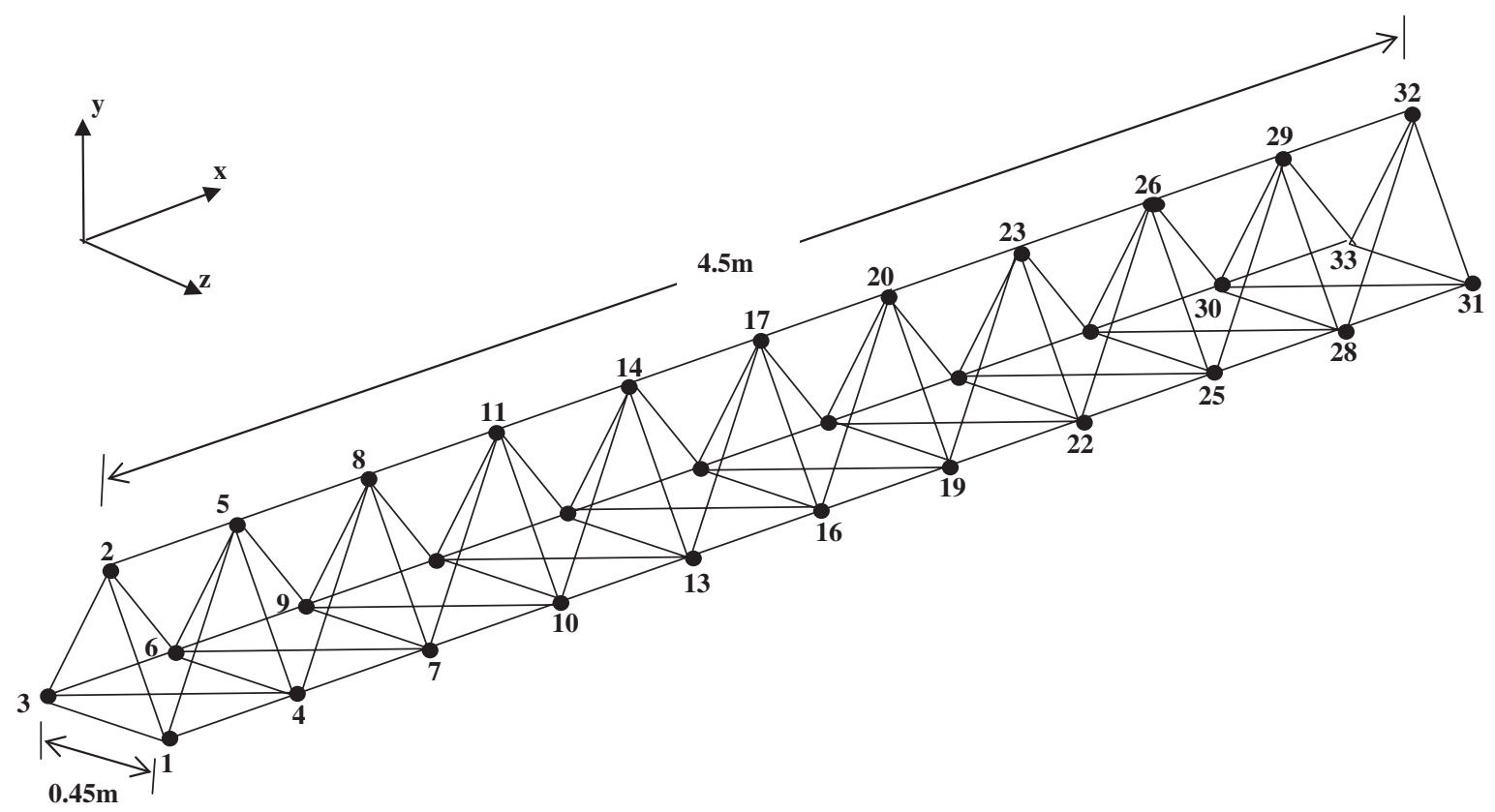

Fig. 1. The numbering system for the joints of the satellite boom structure model.

\section{The base-line experiment}

The experimental set-up for the boom structure is shown in Fig. 3 where the structure is suspended from the ceiling by equal length strings. This approximates free-free boundary conditions, which were chosen here as they are much easier to achieve in practice and the results are as valid as the real-life fixed-free condition. A force transducer (B\&K 8200) was used in conjunction with a shaker (LDS V201) that was screwed to the structure. Joint 4 with coordinates, $0.45,0.0,0.0 \mathrm{~m}$ was chosen as the input force location. The response was measured at the location of one of the end joints, joint 32, with coordinates, 4.5, 0.3897, $-0.225 \mathrm{~m}$, see Fig. 1. A random input force spanning $150-250 \mathrm{~Hz}$, generated by the analyser was supplied to the shaker via an amplifier (type TPA100-D). The translational responses of the structure in the $x$-, $y$ - and $z$ directions at the end three joints (i.e., 31, 32 and 33) were measured by using a Bruel \& Kjaer triaxial accelerometer (type 4326 A) via charge amplifiers (B\&K 2635) whose gains were also recorded for later use. As the accelerometer weighs around $13 \mathrm{~g}$, during any joint measurement, the other two end joints had masses of equal weight attached to them. The measured accelerations were integrated to obtain the corresponding velocities. These outputs were fed back to the analyser.

\section{Results}

Fig. 4 shows the experimental forced response of the structure at joints 31,32 and 33 and in all three translational directions for the frequency bandwidth of $150-250 \mathrm{~Hz}$, compared with the 


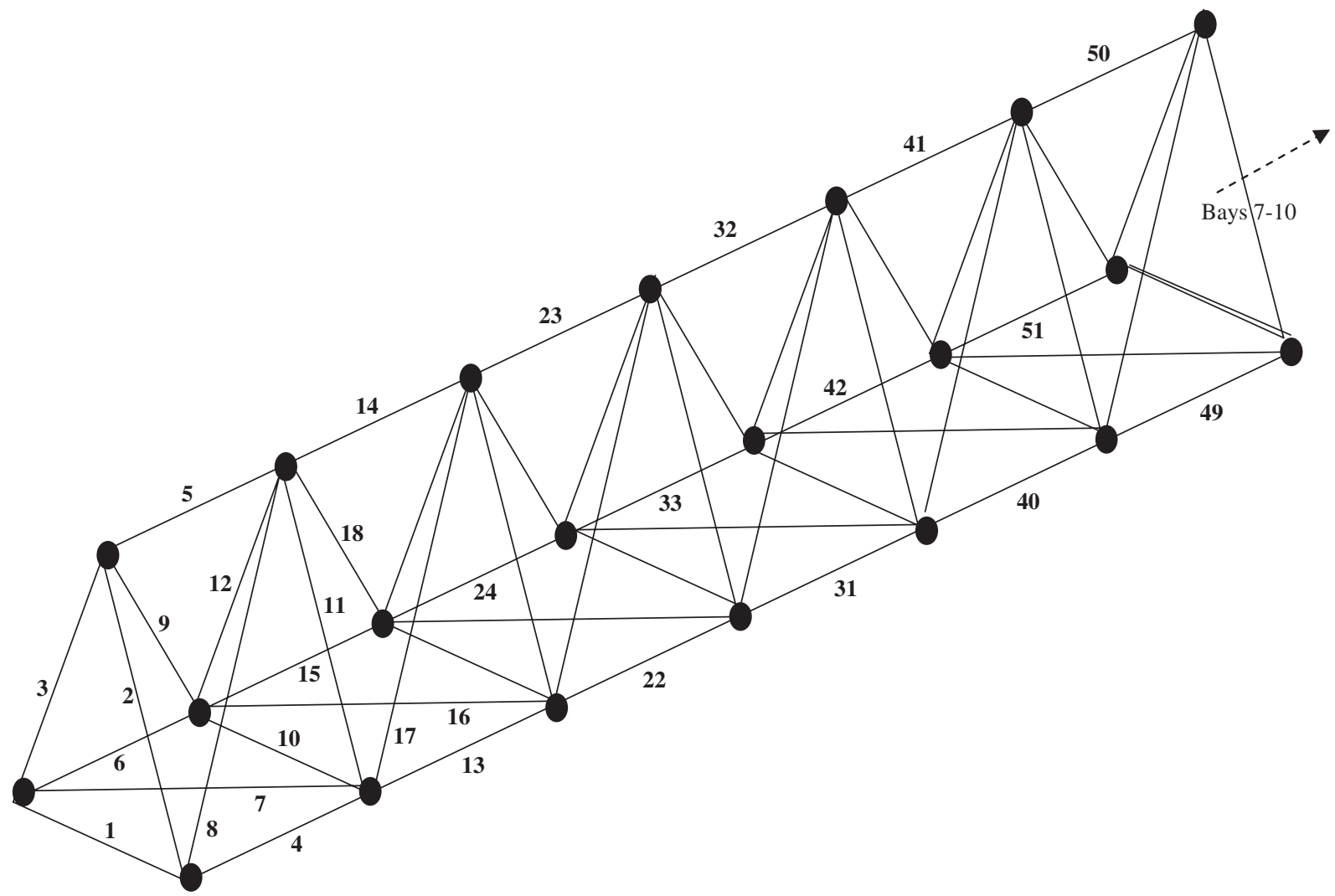

Fig. 2. The numbering system for the beam elements of the satellite boom structure.

predicted results obtained using receptance methods [6]. The graphs in Fig. 4 show reasonable agreement between the experimental results and the theoretical curves. This implies that the theoretical model is capable of predicting the vibrational behaviour of the satellite boom structure and can be used to search for optimum actuator locations in order to reduce its vibration transmission levels.

\section{Active vibration control (AVC)}

In order to reduce the vibration of the boom at its three right end joints, AVC was applied. Here, the AVC is taken to be a feed-forward implementation with each frequency considered separately (this is most suited to tonal vibrations and their harmonics) and therefore the problem of non-causality is avoided. Feed-forward control requires a coherent reference of the vibration source that is not subject to feedback from the control actuators. In the experiments, the source of vibration disturbance is a force applied to one of the joints near the base of the structure, while the reference signal is independently available from the source. In most applications of AVC the most commonly used cost functions are based on the square of a quantity (e.g., acceleration or velocity) 


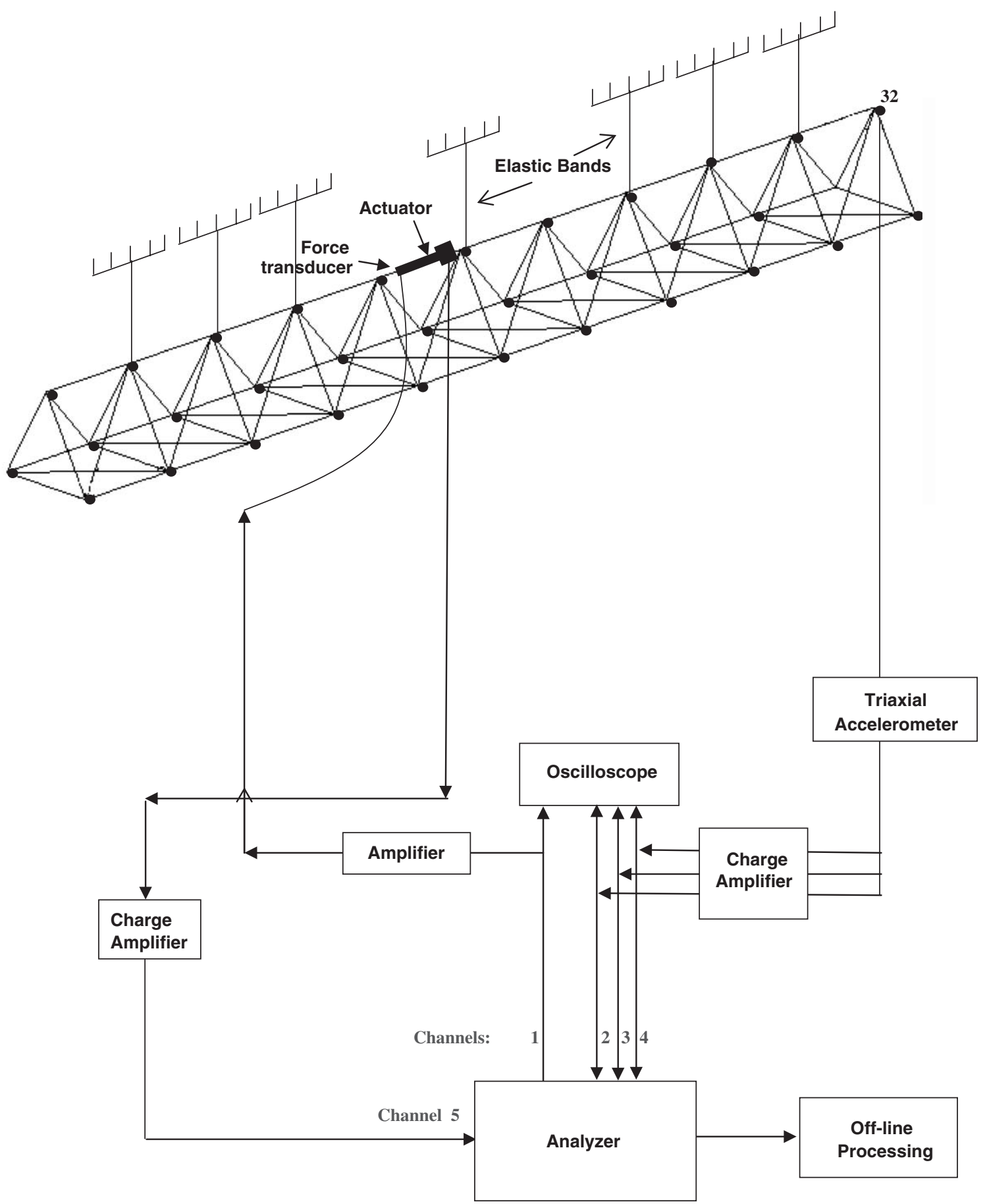

Fig. 3. Experimental set-up for AVC with one actuator on beam 41. 

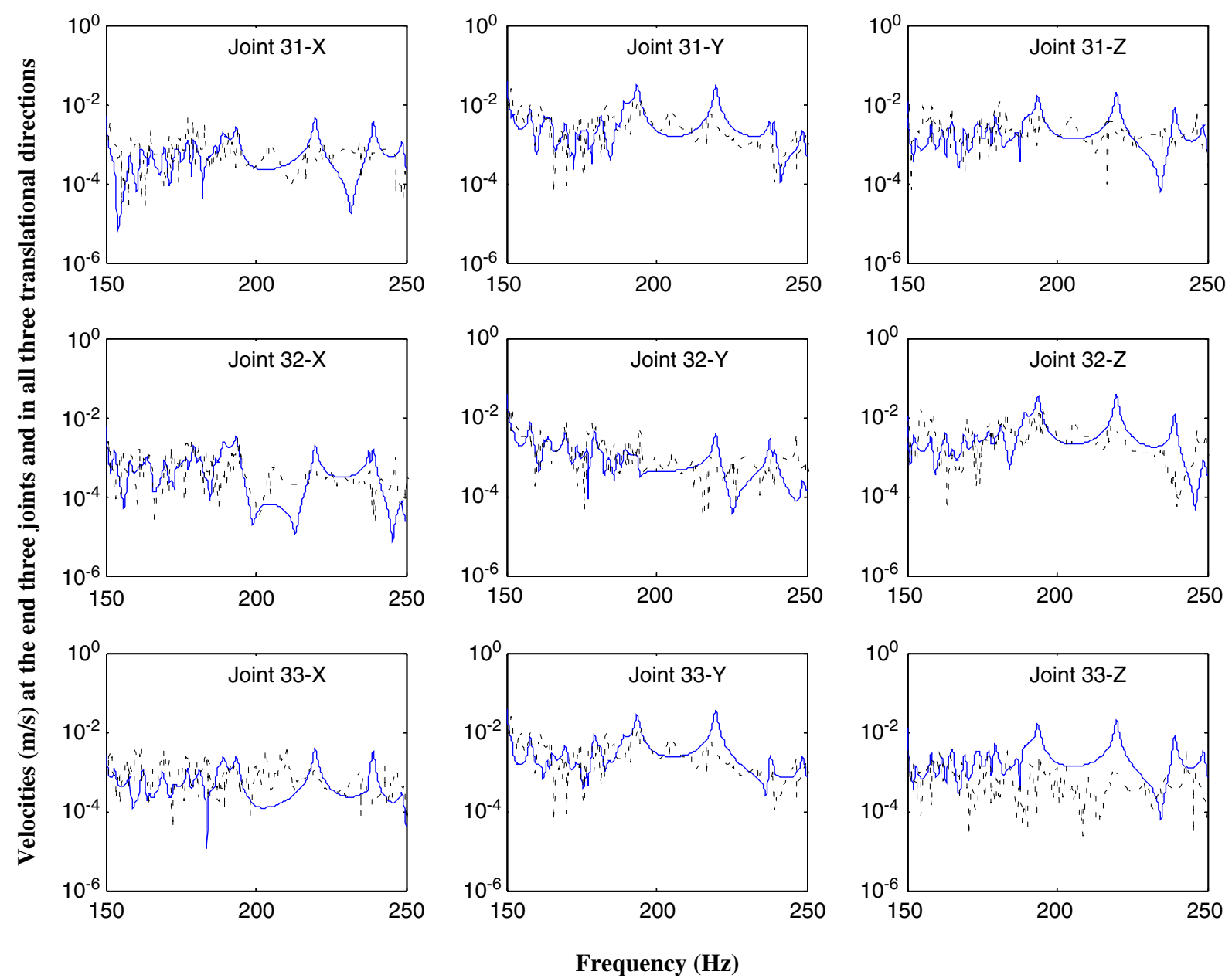

Fig. 4. Experimental and theoretical forced responses of the regular boom structure.

and the formulation of sensible cost functions for this application has been discussed by Anthony and Elliott [7]. More comprehensive control of the structure can be obtained by controlling power, and an example of such a control strategy for a plate is given in Ref. [8]. The use of acceleration can be a good estimate of the power in an infinite beam [9], although it can be very inaccurate close to a structural discontinuity, such as a joint or a source [10]. Controlling power in a structure would normally require the complexity of measuring interbeam coupling forces; however, good estimates of the power in a beam can be achieved using velocity measurements at the beam ends. Furthermore, for a structure comprising of stiff, relatively inflexible beams (or rods) it is sufficient to use only the translational velocity components.

Here, a cost function comprising the translational velocity components at three joints on the structure was used. In this way, the vibration of the "end-face" plane on which the joints are 
located is controlled. The material properties of the actuators are considered in the model. The base vibration (i.e., the primary force) is modelled as a single force of $1 \mathrm{~N}$ applied in the direction of the $y$-axis at one of the joints. The translational velocity components at joints 31,32 and 33 of the structure, at a single frequency, are represented by the velocity vector, $\mathbf{v}$

$$
\mathbf{v}=\left[\begin{array}{lll}
\mathbf{v}^{31} & \mathbf{v}^{32} & \mathbf{v}^{33}
\end{array}\right]^{\mathrm{T}},
$$

which is comprised of three individual joint velocity vectors in the same format. The format of $\mathbf{v}^{31}$ for example comprises the velocity components in the directions $x, y, z$

$$
\mathbf{v}^{31}=\left\{\begin{array}{lll}
v_{x}^{31} & v_{y}^{31} & v_{z}^{31}
\end{array}\right\}^{\mathrm{T}} .
$$

With the AVC operational, the net velocities at the three joints, $\mathbf{v}$, are the result of constructive interference between the velocities resulting from the vibration disturbance (the primary velocity, $\mathbf{v}_{p}$ ) and the velocities resulting from the AVC, $\mathbf{v}_{s}$

$$
\mathbf{v}=\mathbf{v}_{p}+\mathbf{v}_{s} .
$$

The control forces $\mathbf{f}_{s}$ are the quantity controlled by the AVC controller and are applied using double-acting axial actuators in order to reduce the effect of the primary vibration, $\mathbf{v}_{p}$. The secondary velocity vector, which describes the effect of the AVC, can be derived from the secondary control forces by means of a transformed mobility transfer matrix, Y, so Eq. (3) can be written as

$$
\mathbf{v}=\mathbf{v}_{p}+\mathbf{Y} \mathbf{f}_{s} .
$$

$\mathbf{Y}$ is comprised of two matrices, $\mathbf{U}$, the mobility matrix, and $\mathbf{T}$, the transformation matrix, so Eq. (4) can be written as [11]

$$
\mathbf{v}=\mathbf{v}_{p}+\mathbf{U T f}_{s} .
$$

The formats of the above matrices may be illustrated by means of an example. Eq. (6) shows Eq. (5) expanded explicitly for the case of two secondary actuators, where $f_{s_{1}}$ and $f_{s_{2}}$ are the individual forces of the two actuators

$$
\left[\begin{array}{c}
\mathbf{v}^{31} \\
\mathbf{v}^{32} \\
\mathbf{v}^{33}
\end{array}\right]=\left[\begin{array}{c}
\mathbf{v}_{p}^{31} \\
\mathbf{v}_{p}^{32} \\
\mathbf{v}_{p}^{33}
\end{array}\right]+\left[\begin{array}{ll:ll:l}
\mathbf{U}_{1 A}^{31} & -\mathbf{U}_{1 B}^{31} & \mathbf{U}_{2 A}^{31} & -\mathbf{U}_{2 B}^{31} & \ldots \\
\mathbf{U}_{1 A}^{32} & -\mathbf{U}_{1 B}^{32} & \mathbf{U}_{2 A}^{32} & -\mathbf{U}_{2 B}^{32} & \ldots \\
\mathbf{U}_{1 A}^{33} & -\mathbf{U}_{1 B}^{33} & \mathbf{U}_{2 A}^{33} & -\mathbf{U}_{2 B}^{33} & \ldots
\end{array}\right]\left[\begin{array}{lll}
\{\mathbf{1}\} & & \\
& \{\mathbf{1}\} & \\
& & . \\
& &
\end{array}\right]\left[\begin{array}{c}
f_{s_{1}} \\
f_{s_{2}} \\
\cdot \\
.
\end{array}\right] .
$$

Here $\mathbf{U}$ is made up of a number of sub-matrices, each representing the transfer mobility from the force applied at one end of an actuator to all the velocity components at a single joint. For example $\mathbf{U}_{1 A}^{31}$ is

$$
\mathbf{U}_{1 A}^{31}=\operatorname{diag}\left(\begin{array}{lll}
u_{1 A_{x}}^{31} & u_{1 A_{y}}^{31} & u_{1 A_{z}}^{31}
\end{array}\right),
$$

where each component is a single complex transfer mobility. Here, $u_{1 A_{x}}^{31}$, is the transfer mobility from end $A$ of actuator 1 to the velocity in the $\mathbf{x}$-direction at joint 31 . $\mathbf{U}$ is shown partitioned for 
each actuator, where each partition contains sub-matrices representing the transfer mobilities from each end of the actuator to joints 31,32 and 33 . The signs of the sub-matrices for drive end $B$ are negative in order to represent the double-acting operation of the actuators. The transformation matrix, $\mathbf{T}$, is made up of vectors of ones which is given by

$$
\{\mathbf{1}\}=\left\{\begin{array}{llllll}
1 & 1 & 1 & 1 & 1 & 1
\end{array}\right\}^{\mathrm{T}} .
$$

The transformation matrix is required as each secondary control force affects each of the six joint velocities components. T maps each force onto the transfer mobilities relating it to these velocities.

In the application of AVC [12,13], it is usual to minimize the sum of the square of the velocities, i.e., a quadratic function in $\mathbf{f}_{s}$. The result is commonly termed the cost function, $J$, and here is given by

$$
J=\mathbf{v}^{\mathrm{H}} \mathbf{v}
$$

Substituting for $\mathbf{v}$ from Eq. (5), gives

$$
J=f_{s}^{\mathrm{H}} \mathbf{A} f_{s}+f_{s}^{\mathrm{H}} \mathbf{b}+\mathbf{b}^{\mathrm{H}} f_{s}+c,
$$

where

$$
\mathbf{A}=\mathbf{T}^{\mathrm{T}} \mathbf{U}^{\mathrm{H}} \mathbf{U T}, \quad \mathbf{b}=\mathbf{T}^{\mathrm{T}} \mathbf{U}^{\mathrm{H}} \mathbf{v}_{p} \quad \text { and } \quad c=\mathbf{v}_{p}^{\mathrm{H}} \mathbf{v}_{p} .
$$

The minimum value of Eq. (10), $J_{\min }$, is achieved with the optimum vector [14]

$$
\mathbf{f}_{s_{\mathrm{opt}}}=\min (J)=-\mathbf{A}^{-1} \mathbf{b}
$$

and so,

$$
J_{\min }=c-\mathbf{b}^{\mathrm{H}} \mathbf{A}^{-1} \mathbf{b} .
$$

The nominal (uncontrolled) value of Eq. (10) without $\operatorname{AVC}\left(f_{s}=0\right)$ is given by

$$
J_{\text {nom }}=c \text {. }
$$

It is common to use AVC to control a number of frequencies within a specified band. In this case, the optimal secondary force vector and the minimized value of the vibration are found on a frequency-by-frequency basis, as shown above, but then the performance is taken as the arithmetic mean of the vibration at each frequency considered. Hence

$$
\overline{J_{\min }}=\frac{1}{n} \sum_{k=1}^{n} J_{\min }\left(\omega_{L}+(k-1) \Delta \omega\right)
$$

is the average minimized value of the cost function of the frequency band, starting at frequency $\omega_{L}$ and with frequency increment $\Delta \omega . \overline{J_{\text {nom }}}$, the average value of the cost function without $\mathrm{AVC}$, is similarly calculated. The frequency-averaged performance (the attenuation of the mean of the squares of the velocities) is given in decibels by $\alpha$

$$
\alpha=10 \times \log _{10}\left(\overline{\frac{J_{\mathrm{nom}}}{J_{\mathrm{opt}}}}\right) .
$$


When the performance of an AVC system is evaluated over a number of frequencies it is the total control effort that determines the control requirement across the frequency band, $q_{s}$

$$
q_{s}=\sum_{k=1}^{n} \mathbf{f}_{s}^{\mathrm{H}}\left(\omega_{L}+(k-1) \Delta \omega\right) \mathbf{f}_{s}\left(\omega_{L}+(k-1) \Delta \omega\right) .
$$

This parameter is an indication of the electrical power required by the control system using force actuators, and is used for comparative purposes between solutions.

\section{AVC experimental set-up}

The experimental set-up for the active control is illustrated in Fig. 3. The actuators used in these experiments are of preloaded open-loop Piezoelectric Translator (PZT) types that are highresolution linear actuators suitable for static and dynamic applications [14]. They provide submillisecond response and sub-nanometer resolution. The internal spring preload makes them ideal for dynamic applications. The translators are equipped with high reliability multi-layer PZT ceramic stacks protected by an internally spring preloaded non-magnetic stainless-steel case (see Fig. 5 for the dimensions of the PZT). The maximum displacement provided by these actuators is $90 \mu \mathrm{m}$ and they produce pushing and pulling forces of 1000 and $100 \mathrm{~N}$, respectively. Without cables, they weigh around $62 \mathrm{~g}$ and their stiffness is $15 \mathrm{~N} / \mu \mathrm{m}$. The input to the actuator is provided by the analyser through a power amplifier (PI model 790A01). The force applied by the PZT

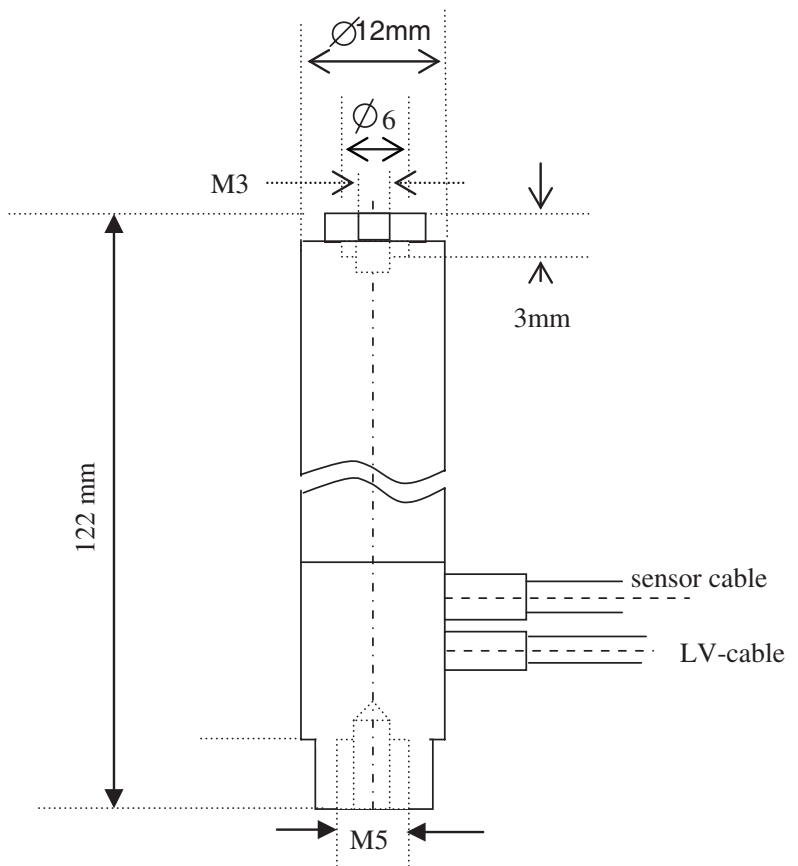

Fig. 5. Dimensions of the preloaded PZT actuator used in the AVC experiments. 
actuator is measured by a force transducer and is measured by the analyser through a charge amplifier. As already noted, the translational responses of the structure in the $x$-, $y$ - and $z$ - directions at the end three joints (i.e., 31, 32 and 33) were measured by using a Bruel \& Kjaer triaxial accelerometer (type 4326A). The measured accelerations were again integrated in order to obtain the corresponding velocities.

\subsection{The optimizer}

Optimization of the type posed here is characterized by having many variables which are related nonlinearly to an objective function that has many peaks and troughs. Since any one configuration is time consuming to analyse, they are very difficult to deal with. When locations are sought for one or two actuators on the structure, an exhaustive search may be conducted. When dealing with three actuators, there are too many possible combinations to work through in this way. Therefore, appropriate optimization strategies must be deployed. The search for methods that can cope with such problems has led to the subject of evolutionary computation. Techniques in this area are characterized by a stochastic approach to the search for improved solutions, guided by some kind of evolutionary control strategy. There three main methods that are currently in use are: (1) simulated annealing [15], where the control strategy is based on an understanding of the kinetics of solidifying crystals; (2) genetic algorithms (GA) [16], where the methods of Darwinian evolution are applied to the selection of "fitter" design; (3) evolutionary programming [17], which is a more heuristic approach to the problem but which has an increasing number of adherents. One of the authors has applied all of these methods to structural problems and found that, for the current case, the GA works best. The GA used here is fairly typical of those discussed in the book by Goldberg [18] but encompasses a number of new ideas that are particularly suited to engineering design problems $[18,19]$. Such methods work by maintaining a pool or population of competing designs which are combined to find improved solutions. In their basic form, each member of the population is presented by a binary string that encodes the variables characterizing the design. The search progresses by manipulating the strings in the pool to provide new generations of designs, hopefully with on average better properties than their predecessors. The processes that are used to seek these improved designs are set up to mimic those of natural selection, hence the method's name.

The most commonly used operations are currently (1) selection according to fitness, i.e., the most promising designs are given a bigger share of the next generation; (2) crossover, where portions of two good designs, chosen at random, are used to form a new design, i.e., two parents "breed" an "offspring"; (3) inversion, whereby the genetic encoding of a design is modified so that subsequent crossover operations affect different aspects of the design; (4) mutation, where small but random changes are arbitrarily introduced into a design. In addition, the number of generations and their size must be chosen, along with a method for dealing with constraints (usually by application of a penalty function).

The algorithm used here works with 12 bit binary encoding. It uses an elitist survival strategy which ensures that the best of each generation always enters the next generation and has optional niche forming to prevent a few moderately successful designs dominating and so preventing wide ranging searches. Two penalty functions are available to deal with constraints. The main parameters used to control the method may be summarized as follows: $N_{\text {gen }}$, the number of 
generations allowed (default 20); $N_{\text {pop }}$, the population size or number of trials used per generation which is therefore inversely related to the number of generations given a fixed number of trials in total (default 250); $P$ [best], the proportion of the population that survive to the next generation (default 0.8$) ; P$ [cross], the proportion of the surviving population that are allowed to breed (default 0.8$) ; P$ [invert], the proportion of this population that have their genetic material re-ordered (default 0.5); $P$ [mutation], the proportion of the new generation's genetic material that is randomly changed (default 0.01); a proportionality flag, which selects whether the new generation is biased in favour of the most successful members of the previous generation or alternatively if all $P$ [best] survivors are propagated equally (default TRUE); the penalty function choice.

When using the GA to explore large design spaces with many variables, it has also been found that the method must be prevented from being dominated by a few moderately good designs which prevent further innovation. A number of methods have been proposed to deal with this problem and the one used here is based on MacQueen's Adaptive KMEAN algorithm [20] which has been applied with some success to multi-peak problems [21]. This algorithm subdivides the population into clusters that have similar properties. The members of each cluster are then penalized according to how many members the cluster has and how far it lies from the cluster centre. It also, optionally, restricts the crossover process that forms the heart of the GA, so that large successful clusters mix solely with themselves. This aids convergence of the method, since radical new ideas are prevented from contaminating such sub-pools.

The version of the algorithm used here is controlled by the following; $D_{\min }$, minimum nondimensional Euclidean distance between cluster centres, with clusters closer than this being collapsed (default 0.05); $D_{\max }$, maximum non-dimensional Euclidean radius of a cluster, beyond which clusters subdivide (default 0.2 ); $N_{\text {clust }}$, the initial number of clusters (default 25); $N_{\text {breed }}$, the minimum number of members in a cluster before exclusive in breeding within the cluster takes place (default 5) and $\alpha$, the penalizing index for cluster members which determines how severely members sharing an over crowded niche will suffer, with small numbers giving greater penalty (default 0.5), i.e., the objective functions of members of a cluster of $m$ solutions are scaled by: $M^{\min (\alpha, 1)}\left[1-\left(E / D_{\max }\right)^{\alpha}\right]+\left(E / D_{\max }\right)^{\alpha}$, where $E$ is the Euclidean distance of the member from its cluster centre (which is always less than $D_{\max }$; moreover, when $E=D_{\max }$ no penalty is applied). In addition, the implementation of the GA used here allows the solution of individual members of the population to be run in parallel if a multiple processor computer or cluster of computers is available.

\section{Selection of optimal actuator positions on the boom structure}

Due to dynamic mechanical coupling between the primary forces and the secondary actuators and their effects at the beam end, the success of AVC heavily depends on the actuator positions in the structure. In this study, the average energy level taken over the frequency bandwidth $150-250 \mathrm{~Hz}$ with resolution of $5 \mathrm{~Hz}$ (i.e., 21 equally spaced frequency points) was used as the parameter to be minimized. Although a much higher-frequency resolution would be desirable in these calculations, the computational effort required would be prohibited using the available computing facilities. Nevertheless, the use of 21 frequencies captures much of the overall 
behaviour of the structure. The performance improvement of the AVC subsequently corresponds to maximizing the average attenuation of this parameter.

To calculate the cost function and other related optimization parameters, the measured accelerations were integrated to obtain the required velocities. At the same time, the control effort (as given by Eq. (17), which is an indication of the electrical power required by the actuators and has the arbitrary units $\mathrm{N}^{2}$ ) was evaluated for a $1 \mathrm{~N}$ transverse primary input force. Therefore, any increase in the primary force would result in an increase in the control effort proportional to the square of this primary force. It should be noted that the theoretical model used to predict the response of the actively controlled structure has taken into account the passive effects of the actuators.

\subsection{Single secondary force}

The best position for a single actuator was obtained through an exhaustive search since this is quicker than using an optimizer (as there are only 93 beams). The result of the search showed that beam 41 (see again Fig. 2) was the best position for this single secondary force, and therefore an experiment was carried out with the actuator positioned on this beam. The input force signal to the shaker and the actuator was a stepped-sine wave covering the frequency range of $150-250 \mathrm{~Hz}$. The frequency resolution for all measurements was chosen to be $0.03125 \mathrm{~Hz}$. The output of the actuator was measured by a force transducer (B\&K 8200) which was placed between the actuator and the beam. The response to the secondary input force on element 41 at joints 31,32 and 33 in all three directions were measured using the triaxial accelerometer. Having obtained the velocities due to the secondary force and recalling the velocities due to the primary input force, it was then possible to calculate the experimental objective function and subsequently compare it with the theoretical predictions. The experimental results and their theoretical counterparts are shown in Fig. 6. These results are based on the uncontrolled cost function being the sum of the velocity squares at the end three joints averaged over the frequency range of $150-250 \mathrm{~Hz}$, i.e.,

$$
J=\sum_{150 \mathrm{~Hz}}^{250 \mathrm{~Hz}} \sum_{j=31}^{33}\left(\mathbf{v}_{j x}^{2}+\mathbf{v}_{j y}^{2}+\mathbf{v}_{j z}^{2}\right), \quad j=\text { joint. }
$$

It should be noted that the experimental results show many more resonant peaks than the theoretical plots. This arises because of the limitations of the actuators used, which are not entirely rigid and which introduce asymmetry into the structure causing many more resonance peak to occur. Nonetheless, the damping models chosen are clearly consistent as evidenced by the resonant peaks widths seen in the figure. Moreover, despite this noise, the search method used still allows low-noise design to be produced. Table 1 lists the five best positions that have computationally been obtained for one actuator together with five randomly positioned actuators. The latter five results are included to demonstrate that the selected position for the actuator is optimal. These results suggest that the best positions for the actuator always occur on one of the non-diagonal connecting elements.

Moreover, the optimum position is on the middle bay closer to the primary force (i.e., the 5th bay). This implies that within the bandwidth considered, the most effective control with one actuator is obtained by blocking the wave propagation path along the boom structure. For these 


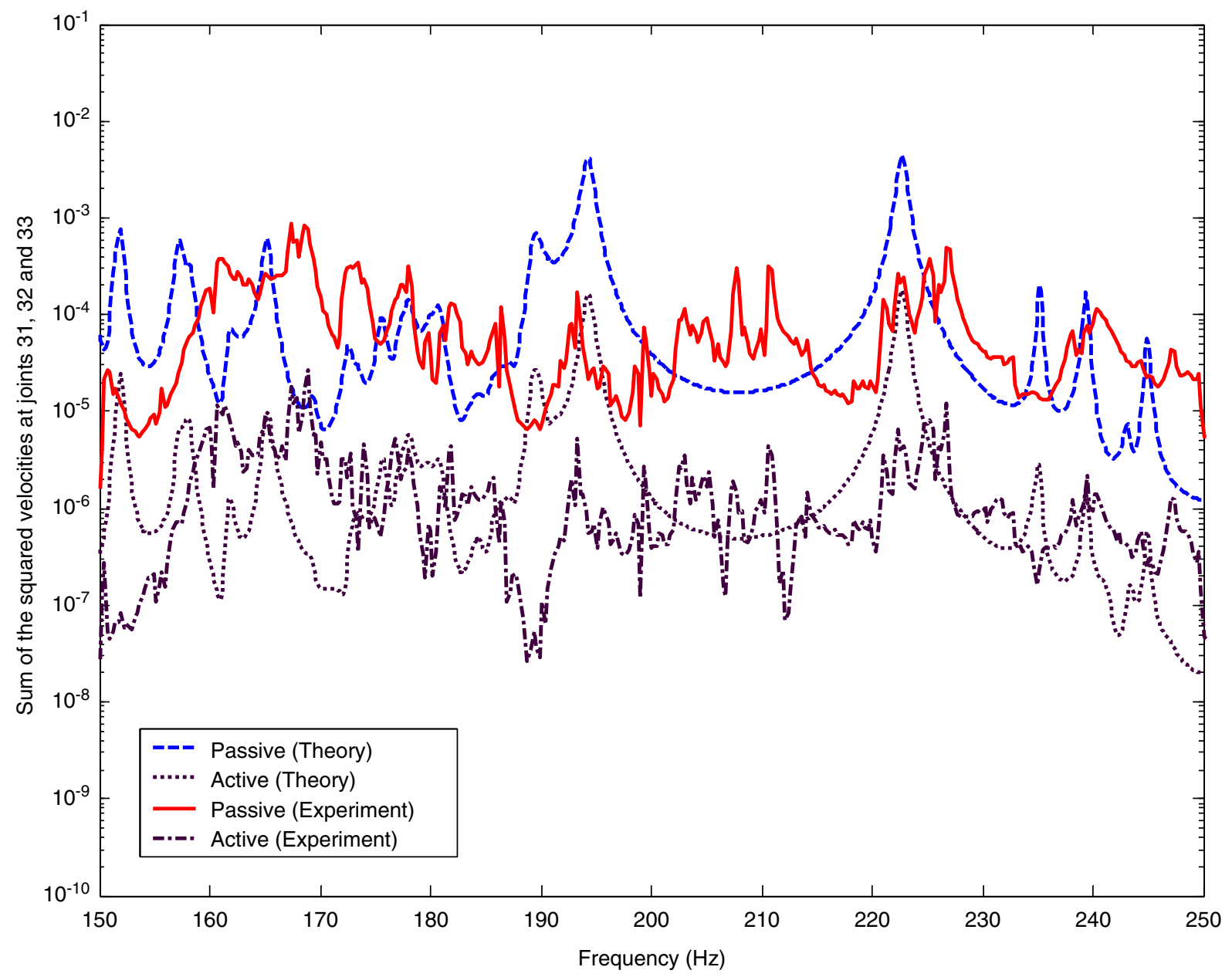

Fig. 6. Theoretical and experimental passive and simulated active objective functions with a single actuator positioned on beam 41 .

actuator positions, the best achievable values of attenuation range from 10.8 to $15.1 \mathrm{~dB}$ with total control effort, given by Eq. (17), ranging from $3.01 \times 10^{5}$ to $4.64 \times 10^{5}$. It can be seen that the secondary forces are very large compared with the primary unit force. This may be explained by the fact that the secondary forces are acting along the structure (i.e., into a high impedance) whereas the primary force acts transverse to the structure (i.e., into a low impedance). Fortunately, the minimum control effort corresponds to the maximum attenuation that is obtained by the optimum actuator position 41 . The five randomly selected actuator positions are also listed in the table mainly as counter examples in order to show that the selected beam number of 41 is the optimal position. The predicted degree of attenuation obtained by placing the actuator on this beam is $15.1 \mathrm{~dB}$. Therefore, the experimental attenuation value of $16.6 \mathrm{~dB}$ that is achieved is slightly higher than the predicted value. 
Table 1

The five best and five random positions for a single actuator

\begin{tabular}{lcc}
\hline Actuator positions & Attenuation $(\mathrm{dB})$ & Control effort $\left(\mathrm{N}^{2}\right)$ \\
\hline Top best actuator locations & & \\
41 & 15.1 & $3.01 \times 10^{5}$ \\
50 & 13.8 & $4.64 \times 10^{5}$ \\
40 & 12.9 & $3.38 \times 10^{5}$ \\
49 & 12.6 & $4.31 \times 10^{5}$ \\
31 & 10.8 & $4.06 \times 10^{5}$ \\
Random actuator locations & & \\
11 & 3.0 & $4.05 \times 10^{5}$ \\
16 & 5.3 & $3.76 \times 10^{5}$ \\
49 & 3.7 & $3.93 \times 10^{5}$ \\
62 & 7.6 & $4.57 \times 10^{5}$ \\
79 & 3.7 & $4.26 \times 10^{5}$ \\
\hline
\end{tabular}

Table 2

The five best and five random locations for 2 actuators

\begin{tabular}{llc}
\hline Actuator positions & Attenuation $(\mathrm{dB})$ & Control effort $\left(\mathrm{N}^{2}\right)$ \\
\hline Top best actuator locations & & \\
8,45 & 26.1 & $10.68 \times 10^{5}$ \\
8,53 & 24.8 & $10.66 \times 10^{5}$ \\
20,45 & 24.6 & $17.24 \times 10^{5}$ \\
11,45 & 23.4 & $10.97 \times 10^{5}$ \\
44,8 & 23.0 & $10.42 \times 10^{5}$ \\
Random actuator locations & & \\
53,12 & 12.7 & $6.45 \times 10^{5}$ \\
51,93 & 4.1 & $3.30 \times 10^{5}$ \\
59,44 & 16.4 & $7.48 \times 10^{5}$ \\
47,92 & 7.2 & $6.76 \times 10^{5}$ \\
52,61 & 4.3 & $6.92 \times 10^{5}$ \\
\hline
\end{tabular}

\subsection{Two secondary forces}

In order to find the optimum position for two secondary forces an exhaustive search was once again carried out. The search indicated that beams number 8 and 45 provided the best positions for two actuators acting together. The amount of attenuation obtained by these two actuators is $26.1 \mathrm{~dB}$ which is significantly higher than for one actuator. Table 2 lists the five best computed positions for two actuators. Similar to the one actuator case, the table also demonstrates the optimal value of the actuator position, i.e., when random locations are used much reduced attenuation is achieved. 
The table shows that the best achievable attenuations range from 23.0 to $26.1 \mathrm{~dB}$ and the total control effort required to achieve these attenuations vary from $10.42 \times 10^{5}$ to $17.24 \times 10^{5} \mathrm{~N}^{2}$. The table also demonstrates the optimal values of the actuator position and therefore when random locations are used much reduced attenuations are achieved. To carry out an experiment with new actuator positions involves removing the piezoelectric actuators from their current positions and replacing the original rods in their places and then placing the actuators in their new positions. Repeating this procedure can damage both the structure and the actuators. For this reason, it was decided to carry out further experiments only for the three actuator case.

\subsection{Three secondary forces}

Finally, to find the best positions for three secondary forces, the GA was employed. As a result of an extensive search, the best positions for the three actuators were found to be on beams 23, 60 and 71 . This gave an average of $33.5 \mathrm{~dB}$ attenuation. Here the equivalent active control experiment is not carried out in real time. Instead, a simpler experiment is repeated four times and the response of the structure to the primary force and the three secondary forces are measured separately and the overall $J_{\min }$ is calculated off-line, using Eqs. (13)-(17). Therefore, the experimental set-up for three actuators is the same as when one actuator was used (see again Fig. 3).

As before, the structure was first excited by an external force at joint 4 with coordinates, 0.45 , $0.00,0.00 \mathrm{~m}$, and the response measured using a triaxial accelerometer at the end three joints in $x$-, $y$ - and $z$-directions. Then the external force (i.e., the shaker) was removed and the structure was excited by the three actuators, one at a time. Having measured the responses to the primary and the three secondary forces $J_{\min }$ was calculated using Eq. (14). The theoretical values were again found by the use of the receptance code. Fig. 7 shows the results obtained. The experimental attenuation was found to be $31.7 \mathrm{~dB}$ which is slightly less than the predicted value of $33.5 \mathrm{~dB}$.

When comparing the two sets of data, one should consider the potential sources of error in both experiments and theory. As far as the experiments are concerned, one of the main sources of error is the low signal-to-noise ratio, when the actuators are exciting the structure. Increasing the input gain could have pushed the actuators' outputs into their nonlinear regions and so their outputs were necessarily small. As for the theoretical model, one obvious source of error is the addition of joints and elements (e.g., nuts, screws, force transducers) on the rods where the actuators are placed that are only approximately accounted for in the theoretical model.

\section{Conclusions}

This paper has shown the ability of a receptance code to successfully model the vibrational behaviour of a satellite boom structure over a wide frequency bandwidth. It has also illustrated the kinds of improvements in performance that may be achieved using feed-forward active control. The amount of vibration transmission reduction achieved from one end to the other of a $4.5 \mathrm{~m}$ satellite boom was shown here to be $15.1 \mathrm{~dB}$ with one, $26.1 \mathrm{~dB}$ with two and $33.5 \mathrm{~dB}$ with three secondary forces. Experiments were carried out to confirm these reductions and satisfactory agreements were obtained. 


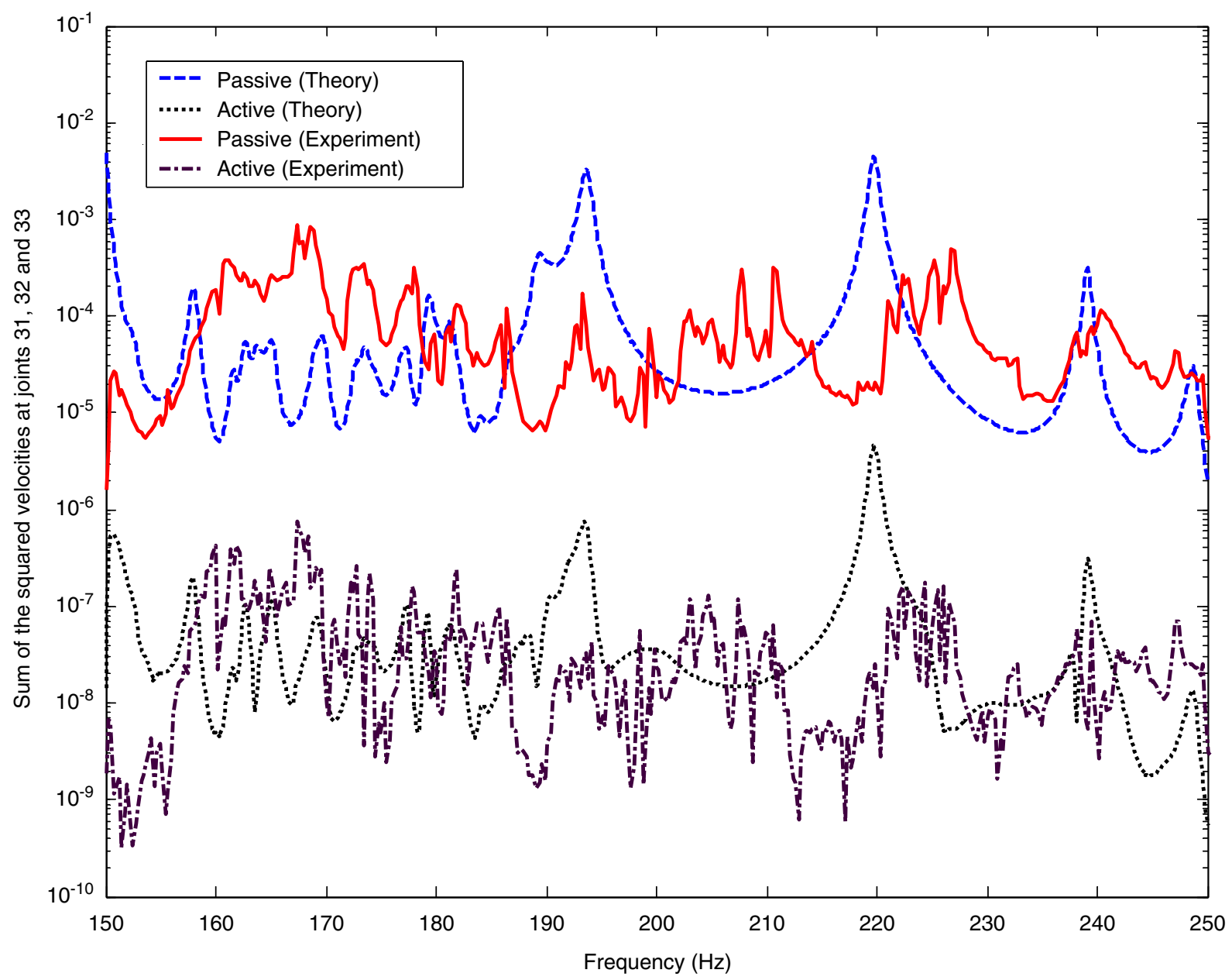

Fig. 7. Theoretical and experimental passive and active objective functions for the regular boom with the actuators positioned on beams 23,60 and 71 .

\section{Acknowledgement}

This work was supported by the EPSRC under Grant Reference GR/M33624 and by Astrium Space.

\section{References}

[1] J.W. Melody, H.C. Briggs, Analysis of structural and optical interactions of the Precision Optical Interferometer, Proceedings of the SPIE 1947 (1993), 44-57.

[2] S.W. Sirlin, R.A Laskin, Sizing of active piezoelectric struts for vibration suppression on a space-based interferometer, Proceedings of the First US/Japan Conference on Adaptive Structures, 1990, pp. 47-63. 
[3] J.W. Melody, et al., Integrated modeling methodology validation using the micro-precision interferometer testbed, Proceedings of the IEEE CDC Conference, Kobe, 1996.

[4] M. Moshrefi-Torbati, A.J. Keane, S.J. Elliott, M.J. Brennan, E. Rogers, The integration of advanced active and passive structural vibration control, Proceedings of VETOMAC-I, October 25-27, 2000, Bangalore, India.

[5] A.J. Keane, The OPTIONS Design Exploration System User Guide and Reference Manual, 1994, http:// www.soton.ac.uk/ ajk/options/welcome.html.

[6] K. Shankar, A.J. Keane, Energy flow predictions in a structure of rigidly joined beams using receptance theory, Journal of Sound and Vibration 185 (1995) 867-890.

[7] D.K. Anthony, S.J. Elliott, Comparison of the effectiveness of minimizing cost function parameters for active control of vibrational energy transmission in a lightly damped structure, Journal of Sound and Vibration 237 (2) (2000) 223-244.

[8] O. Bardou, P. Gardonio, S.J. Elliott, R.J. Pinnington, Active power minimization and power absorption in a plate with force and moment excitation, Journal of Sound and Vibration 208 (1997) 111-151.

[9] M.J. Brennan, S.J. Elliott, R.J. Pinnington, Strategies for the active control of flexural vibration on a beam, Journal of Sound and Vibration 186 (1995) 657-688.

[10] X. Pan, C.H. Hansen, The effect of error sensor location and type on active control of beam vibration, Journal of Sound and Vibration 165 (1993) 497-510.

[11] D.K. Anthony, S.J. Elliott, Robustness of optimal design solutions to reduce vibration transmission in a lightweight 2-d structure, part ii: application of active vibration control techniques, Journal of Sound and Vibration 229 (3) (2000) 529-548.

[12] C.R. Fuller, S.J. Elliott, P.A. Nelson, Active Control of Vibration, Academic Press, London, 1996.

[13] P.A. Nelson, S.J. Elliott, Active Control of Sound, Academic Press, New York, 1992.

[14] PI Catalog, Solutions for Cutting-Edge Technologies, 2002, http://katalog.physikinstrumente.com/links/topicpdfs/ 2001pdfcatalogstart.html.

[15] S. Kipkpatrick, C.D. Gelatt Jr., M.P. Vecchi, Optimization by simulated annealing, Science 220 (4598) (1983) 671-680.

[16] D.E. Goldberg, Genetic Algorithm in Search Optimization and Machine Learning, Addison-Wesley, Cambridge, MA, 1989.

[17] D.B. Fogel, Applying evolutionary programming to selected travelling salesman problems, Cybernetics and Systems 24 (1) (1993) 27-36.

[18] A.J. Keane, Structural design for enhanced noise performance using Genetic Algorithm and other optimisation techniques, in: R.F. Albrecht, C.R. Reeves, N.C. Steele (Eds.), Proceedings of the International Conference on Artificial Neural Nets and Genetic Algorithms, Springer, Innsbruck, 1993, pp. 536-543.

[19] A.J. Keane, Genetic algorithm optimisation of multi-peak problems: studies in convergence and robustness, Artificial Intelligence in Engineering 9 (2) (1995) 75-83.

[20] M.R. Anderberg, Cluster Analysis for Applications, Academic Press, New York, NY, 1975.

[21] X. Yin, N. Germay, A fast genetic algorithm with sharing scheme using cluster methods in multimodal function optimisation, in: R.F. Albrecht, C.R. Reeves, N.C. Steele (Eds.), Proceedings of the International Conference on Artificial Neural Nets and Genetic Algorithms, Springer, Innsbruck, 1993, pp. 450-457. 